\title{
Space-time clustering of childhood leukaemia cases in Karachi, Pakistan
}

\author{
Saeed Akhtar, Tim E Carpenter, Fouzia Ajmal, Shafquat Rozi, Salman Adil
}

J Epidemiol Community Health 2005;59:221-222. doi: 10.1136/jech.2004.022178

$\mathrm{T}$ he incidence rate of childhood leukaemia varies internationally (about $4-40 / 10^{6}$ per year) with the lowest rates being recorded in black African children. ${ }^{1}$ The data from population based studies on the incidence of childhood leukaemia in South Asia, including Pakistan, are lacking. The development of leukaemia has been considered as an infectious process. ${ }^{2}$ However, the epidemiological evidence for the existence of childhood leukaemia virus has been regarded as naive. Also, the question whether childhood leukaemias have a tendency to occur in cluster remains unanswered. ${ }^{3}$ We conducted this study to determine if clustering of cases of childhood leukaemia at diagnosis in time and time and space if observed was more common than could be expected.

\section{METHODS}

Data collection was carried out between March 2002 and November 2002. All children diagnosed between 1 January 1995 and 30 May 2002 as having any form of leukaemia before their 15th birthday and were born and resident of Karachi, were included in the study. Cases were identified from two tertiary care hospitals and data collected from medical records and by interviewing the parents of case children at their homes. For the residence of each case, geographical coordinates were also recorded and subsequently converted to a kilometre grid.

The Scan test was used to investigate temporal clustering by testing moving windows of several different widths from one to six months. ${ }^{4}$ The Knox method for detecting space and time clustering was used. ${ }^{5}$ The possible pairs considered are $n(n-1) / 2$ pairs for $n$ observed cases. Contingency tables were examined for combinations of six critical time ( $1-6$ months) intervals and 13 critical distance $(1-7 \mathrm{~km})$ intervals. For each of the chosen combinations of temporal-spatial location, the probability of actually observing as many or more paired cases of childhood leukaemia assuming a Poisson distribution was determined using an iterative simulation process. The statistically significant selected space-time combinations were screened with 99 iterations and those with $\mathrm{p}<0.1$ were more closely examined with 999 iterations.

\section{RESULTS}

During the 89 month study period from 1 January 1995 to 30 May 2002, 142 cases of childhood leukaemia were diagnosed at two tertiary care hospitals in Karachi. The results of the Scan test showed that a maximum of seven cases of childhood leukaemia were diagnosed during the time window of one month period from 11 October 1999 to 10 November 1999, which was significantly $(p=0.001)$ more than the expected number of cases (1.6) predicted for one month period. Significant $(\mathrm{p}<0.001)$ temporal clustering was also observed for time windows of two to six months. Spatial and temporal clustering of cases of childhood leukaemia was examined using the Knox test. As is evident from ratios of observed to expected number of case pairs within specified critical times and critical distances, there is a consistent tendency of clustering of childhood leukaemia cases within critical limits of 3-5 km and 30 days and 3-6 km and 60120 days (fig 1). The most significant clustering occurred when critical time was 90 days and critical distance was $6 \mathrm{~km}$. For 142 cases in this study, 10011 case pairs were available for analysis. Of these total case pairs, 244 case pairs occurred within these critical limits of one another. These 244 case pairs were significantly $(p=0.02)$ higher than 222 case pairs expected to occur within critical time-space separation, if the spatial and temporal distribution of cases of childhood leukaemia were independent.

\section{DISCUSSION}

The results of this analysis provided evidence that cases of childhood leukaemia tended to occur in close proximity to

\section{Key points}

- Significant space-time clusters of childhood leukaemia were recorded.

- Findings strengthen the notion that common infection of high virulence and low pathogenecity may be involved in the aetiology of childhood leukaemia.

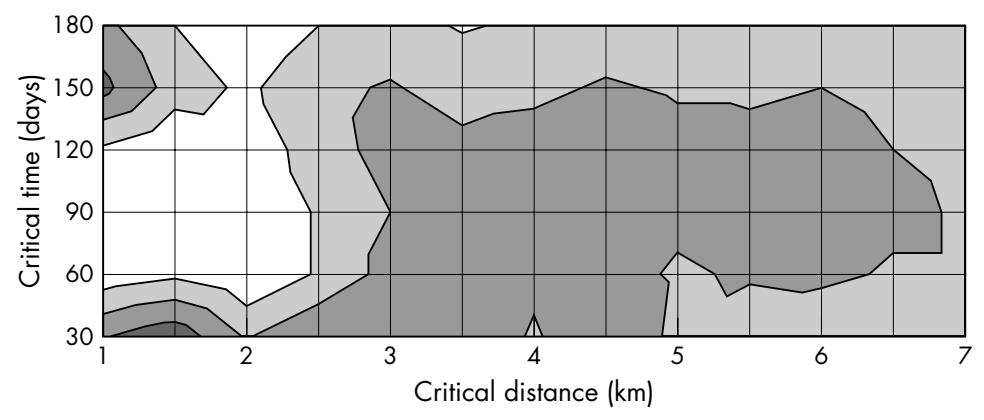

Figure 1 Plot of ratios of observed to 1.15-1.25 expected number of pairs of childhood 1.05-1.15 leukaemia cases located within various $0.95-1.05$ critical time (30-180 days) and 0.85-0.95 distance $(1-7 \mathrm{~km})$ of each other in distance $(1-7 \mathrm{~km})$ of each other in
Karachi, Pakistan, 1 January 1995 to 30 May 2002. 


\section{Policy implications}

In conjunction with cumulative evidence, this study shows that childhood leukaemia may be an infrequent response to infection of high virulence and low pathogenecity. Future research however, is needed to ascertain this contention.

each other in time and time and space with an excess of 22 pairs over the random expectation within $6 \mathrm{~km}$ and 90 days of each other. The space-time clustering has also been shown with regard to residence at diagnosis for 13351 cases of childhood leukaemia diagnosed during 1980-89 in defined geographical regions in 17 other countries. ${ }^{6}$ The results of this study strengthen the notion that a common infection of high virulence and low pathogenecity may be involved in the aetiology of childhood leukaemia.

\section{Authors' affiliations}

S Akhtar, F Ajmal, S Rozi, Department of Community Health Sciences, Aga Khan University, Karachi, Pakistan
S Akhtar, T E Carpenter, Department of Medicine and Epidemiology, University of California, Davis, USA

S Adil, Department of Pathology and Microbiology, Aga Khan University

Funding: this study was funded by the Department of Community Health Sciences, Aga Khan University, Karachi

Conflicts of interest: none declared.

Correspondence to: Dr S Akhtar, Department of Community Health Sciences, Aga Khan University, Karachi, 74800, Pakistan; saeed. akhtar@aku.edu

Accepted for publication 5 July 2004

\section{REFERENCES}

1 Parkin DM, Stiller CA, Draper GJ, et al. The international incidence of childhood cancer. Int J Cancer 1988:42:511-20.

2 Greaves M. Speculations on the cause of childhood acute lymphoblastic leukemia. Leukemia 1988;2:120-25.

3 Doll R. The epidemiology of childhood leukemia. J R Stat Soc 1989:152:341-51.

4 Naus Jl. The distribution of the size of maximum cluster of points on the line. J Am Stat Assoc 1965;60:532-8.

5 Knox EG. Epidemiology of childhood leukaemia in Northumberland and Duham. Br J Prev Soc Med 1964;18:17-24.

6 Alexander FE, Boyle P, Carli PM, et al. Spatial clustering of childhood leukaemia: summary results from the EUROCLUS project. $\mathrm{Br} J$ Cancer 1998;77:818-24. 